ELOHI

Peuples indigènes et environnement

5-6 | 2014

Ressources du vivant

\title{
La régulation de la circulation des savoirs autochtones en France et au Canada : entre immobilisme étatique et dynamisme infra- étatique
}

Thomas Burelli

\section{(2) OpenEdition \\ Journals}

Édition électronique

URL : http://journals.openedition.org/elohi/769

DOI : 10.4000/elohi.769

ISSN : 2268-5243

Éditeur

Presses universitaires de Bordeaux

Édition imprimée

Date de publication : 1 janvier 2014

Pagination : 151-170

ISBN : 979-10-300-0021-4

ISSN : 2431-8175

Référence électronique

Thomas Burelli, « La régulation de la circulation des savoirs autochtones en France et au Canada entre immobilisme étatique et dynamisme infra-étatique », ELOHI [En ligne], 5-6 | 2014, mis en ligne le 01 janvier 2015, consulté le 03 mai 2019. URL : http://journals.openedition.org/elohi/769 ; DOI : 10.4000/elohi.769 


\section{La régulation de la circulation des savoirs autochtones en France et au Canada : entre immobilisme étatique et dynamisme infra-étatique}

\section{THOMAS BURELLI}

Université d'Ottawa

«Quand l'État ne réglemente pas, d'autres s'empressent de le faire à sa place.

Le vide laissé par l'État est rapidement comblé. Ce qui importe [...] est de savoir qui réglemente à la place de l'État, pour quels motifs ou intérêts, selon quels procédés et avec quelles conséquences ».

(Rocher 108)

La notion de savoirs traditionnels ${ }^{1}$ (ou savoirs autochtones) est aujourd'hui reconnue et/ou discutée au sein de nombreuses enceintes internationales dans les domaines de la culture (UNESCO), du droit des peuples autochtones (Nations Unies), de la protection de l'environnement (Convention sur la Diversité Biologique), ou encore de la propriété intellectuelle (Organisation Mondiale de la Propriété Intellectuelle - OMPI).

Les savoirs traditionnels ont ainsi fait l'objet de développements dans le cadre de la Convention pour la sauvegarde du patrimoine culturel immatériel adoptée en

1. Les savoirs traditionnels constituent une des composantes du patrimoine culturel immatériel autochtone et sont à distinguer des expressions culturelles traditionnelles. Il n'existe pas de définition communément admise de la notion de savoirs traditionnels. Il est néanmoins possible de les définir de manière générale comme les connaissances, innovations et pratiques détenues par certains groupes - les communautés autochtones et locales - dans des domaines aussi variés que l'agriculture, la médecine, en passant par les techniques de chasse, les savoirs culinaires ou encore l'artisanat traditionnel (Berkes 3, Sambuc 60, 79). 
2003. Les États signataires se sont engagés dans ce texte à respecter «le patrimoine culturel immatériel des communautés, des groupes et des individus concernés ${ }^{2}$ ", ainsi quà engager des mesures pour la sauvegarde de ce patrimoine 3 .

La déclaration des Nations Unies sur les Droits des peuples autochtones reconnait, elle, un droit de contrôle et de gestion du patrimoine culturel immatériel au bénéfice des Autochtones :

Les peuples autochtones ont le droit de préserver, de contrôler, de protéger et de développer leur patrimoine culturel, leur savoir traditionnel et leurs expressions culturelles traditionnelles ainsi que les manifestations de leurs sciences, techniques et culture, y compris leurs ressources humaines et génétiques, leurs semences, leur pharmacopée, leur connaissance des propriétés de la faune et de la flore, leurs traditions orales, leur littérature, leur esthétique, leurs sports et leurs jeux traditionnels et leurs arts visuels et du spectacle. Ils ont également le droit de préserver, de contrôler, de protéger et de développer leur propriété intellectuelle collective de ce patrimoine culturel, de ce savoir traditionnel et de ces expressions culturelles traditionnelles ${ }^{4}$.

Cependant, le premier traité ayant abordé la question des savoirs traditionnels associés à l'environnement et, aujourd'hui, un des forums les plus actifs concernant cette question, reste la Convention sur la Diversité Biologique (CDB). C'est en effet en 1992 dans cette convention, adoptée lors du sommet de la terre à Rio, que la notion apparaît pour la première fois dans un traité à valeur contraignante. L'objet principal de la CDB est la conservation et l'utilisation durable de la biodiversité. Les savoirs traditionnels n'apparaissent dans ce texte qu’à l'article $8(\mathrm{j})^{5}$. Ils ont été inclus dans le texte du fait de leur intérêt pour la conservation. En effet, les parties à la convention ${ }^{6}$ ont défini les communautés autochtones et locales comme détentrices de savoirs dont l'utilisation pourrait concourir à la réalisation des objectifs de la CDB. Il s'agissait aussi d'encourager les États à la mise en place de dispositifs de partage juste et équitable des avantages découlant de l'utilisation de ces savoirs. Au sens de la CDB, les savoirs traditionnels sont donc des connaissances, innovations et pratiques dont sont dépositaires certaines catégories d'acteurs (les communautés autochtones et

2. Article ${ }^{\text {er }}$, Convention pour la sauvegarde du patrimoine culturel immatériel, du 17 octobre 2003, disponible à l'adresse suivante : http://unesdoc.unesco.org/images/0013/001325/132540f.pdf, page consultée le 10 octobre 2014.

3. Ibid.

4. Article 31 de la Déclaration des Nations Unies sur les droits des peuples autochtones (2007).

5. Convention sur la Diversité Biologique, 1992. Disponible en ligne à l'adresse suivante : https://www.cbd.int/doc/legal/cbd-fr.pdf, page consultée le 10 octobre 2014.

6. États signataires (note des éditeurs). 
locales, sans que celles-ci ne soient définies par le texte). En 2010, le protocole de Nagoya a été adopté avec pour objectif de compléter la CDB et de proposer un cadre contraignant pour les États. Si le texte apporte peu de nouveautés d'un point de vue conceptuel ${ }^{7}$, il approfondit néanmoins les obligations des États en ce qui concerne la circulation des savoirs traditionnels, puisqu'il est maintenant reconnu que le consentement préalable des communautés doit être obtenu pour l'accès à leurs savoirs, ce qui nétait pas prévu par la $\mathrm{CDB}^{8}$.

Cependant, malgré cette constellation de traités ${ }^{9}$ et l'arsenal juridique international existants, beaucoup d'États ne sont pas parvenus jusqu'à présent à adopter de cadres spécifiques de protection des savoirs traditionnels. C'est notamment le cas de la France et du Canada.

En effet, les traités internationaux adoptés confient en général aux États le rôle dévaluer la pertinence d'une action ${ }^{10}$ ainsi que le choix des moyens à mettre en œuvre. Or beaucoup d'entre eux semblent avoir des difficultés à appréhender les enjeux en cause et à développer des réponses juridiques innovantes.

Ils semblent, néanmoins, toujours convaincus de l'importance du droit international et déploient une énergie qui apparaît inversement proportionnelle à leur inaction nationale pour la négociation de normes internationales.

À titre d'illustration, la CDB a tenu 11 conférences des parties depuis 1994 et a adopté 1033 documents dans des domaines divers, ainsi, que 332 décisions. Lors du sommet de Nagoya en 2010 environ 18000 participants se sont déplacés. Lors de la conférence des parties suivante organisée à Hyderabad (Inde) en 2012, 170 pays étaient présents et 15000 délégués inscrits.

De manière parallèle aux négociations internationales, les États continuent aussi de déployer des moyens humains et financiers considérables afin de

7. Pire, le Protocole procède à certaines modifications sémantiques dont il est difficile de préjuger des effets mais qui pourraient être interprétées de manière à restreindre le champ de certains concepts. Ainsi alors que la CDB évoque dans son article 8 (j) « les connaissances, innovations et pratiques des communautés autochtones et locales », le Protocole évoque lui seulement « les connaissances traditionnelles détenues par les communautés autochtones et locales » sans que soit précisée si ces deux notions doivent être entendues comme synonymes (Burelli 2012a : 52).

8. Article 7, Protocole de Nagoya, 15 octobre 2010, disponible à l'adresse suivante : http://www.cbd.int/abs/doc/protocol/nagoya-protocol-fr.pdf, page consultée le 10 octobre 2014 .

9. Sans compter le projet de traité en cours de négociation à l'OMPI, disponible à l'adresse suivante : http://www.wipo.int/edocs/mdocs/tk/fr/wipo_grtkf_ic_24/wipo_ grtkf_ic_24_facilitators_document_rev_2.pdf, page consultée le 10 octobre 2014.

10. Notamment, de répondre à la question de savoir s'ils considèrent que des communautés autochtones et locales détentrices de savoirs se trouvent sur leur territoire. 
déterminer l'opportunité et la faisabilité d'une action nationale. Ainsi le gouvernement canadien a lancé plusieurs séries de consultations sur la question (Environnement Canada 2005 : 5-6 ; Gouvernement du Canada 2005 : 19 et suivantes ; Gouvernement du Canada et Gouvernement du Nouveau-Brunswick 2006). Elles ont mené à la rédaction d'un document explicatif et consultatif en vue de l'adoption éventuelle d'un cadre fédéral de reconnaissance et de protection spécifique des savoirs traditionnels (Environnement Canada 2009). Jusqu’à présent ces travaux n’ont été suivis d'aucune politique ou réglementation spécifique et globale. De son côté, la France a lancé en 2009 une étude sur la pertinence et la faisabilité de la mise en œuvre d'un dispositif d'APA portant sur les ressources génétiques et les savoirs traditionnels dans les territoires de l'outremer (Fondation pour la Recherche sur la Biodiversité 2011).

Les démarches entreprises, tant au niveau international quau niveau national, constituent, sans conteste, des initiatives positives de la part des États. Cependant deux questions se posent : d'une part, la mise en œuvre au niveau national nécessite-t-elle l'adoption d'un corpus de textes juridiques internationaux exhaustifs et détaillés ? D’autre part, les autorités publiques sont-elles les seules autorités légitimes et capables de réguler la circulation des savoirs traditionnels, notamment par l'adoption de cadres réglementaires?

Les situations d'inaction étatique actuellement observables en France et au Canada nous conduisent à proposer une réponse négative à ces deux questions et à constater l'existence d'un foisonnement normatif au niveau infra-étatique. En effet, les individus et certaines institutions se montrent très actifs et font preuve, dans certains cas, d'une grande créativité pour la production de cadres de régulation de la circulation des savoirs autochtones. Cependant, trop souvent, pour diverses raisons, ces pratiques ou dispositifs passent inaperçus alors qu'ils pourraient être d'un grand intérêt pour les États en leur servant de source d'inspiration, ou encore, pour les convaincre de s'abstenir d'intervenir lorsque cela n'est pas nécessaire.

Nous proposons, dans cet article, de passer en revue un certain nombre de stratégies et d'outils développés et disponibles, à ce jour, au niveau local en France et au Canada, pour la gestion de la circulation des savoirs traditionnels. Nous laissons volontairement de côté les solutions basées sur la propriété intellectuelle dont on a souvent souligné le fait qu'elles constituent des alternatives intéressantes mais insuffisantes eu égard aux attentes des communautés autochtones et aux spécificités du patrimoine culturel immatériel (Burelli 2012c : 152-154). Nous souhaitons démontrer, au contraire, qu'il existe des alternatives crédibles aux outils classiques de la propriété intellectuelle. En d’autres termes qu'il est possible de faire preuve de créativité et de proposer des principes et des cadres originaux de collaboration entre chercheurs et autochtones. 
Les stratégies ou dispositifs que nous allons présenter ne sont pas exclusifs l'un de l'autre, au contraire, dans certains cas, ils peuvent communiquer et interagir. Nous ne prétendons évidemment pas à l'exhaustivité, mais nous souhaitons simplement offrir un aperçu du foisonnement normatif observable sur le terrain, et pourtant souvent sous-estimé, sinon ignoré. Nous souhaitons aussi démontrer qu'il n'existe pas de vide juridique dans le champ de la gestion des savoirs traditionnels mais qu'au contraire des principes et des pratiques existent, ou ont émergé, pour pallier l'absence de mesures étatiques. Nous souhaitons ainsi montrer que les collaborations entre autochtones et chercheurs ne sont pas restées figées dans un modèle d'exploitation abusive (Smith), mais plutôt que les règles et les pratiques ont évolué dans certains cas et contextes. En conséquence de quoi, il pourrait être sérieusement envisageable de mettre en œuvre le nouveau paradigme international sans nécessairement avoir à attendre une action de la part des États.

Nous présenterons donc, successivement, les protocoles communautaires et le droit coutumier (1), l'adoption de cadres éthiques et de protocoles de recherche (2), le développement de pratiques contractuelles (3) et, enfin, lémergence d'institutions de gestion des savoirs (4).

\section{Les protocoles communautaires et le droit coutumier}

Les savoirs traditionnels constituent un patrimoine immatériel dont le contrôle est, a priori, plus aisé pour les communautés que le contrôle du patrimoine culturel matériel ou encore de la terre. En tout cas, en ce qui concerne la diffusion initiale des savoirs non divulgués à l'extérieur de la communauté ${ }^{11}$. Les savoirs traditionnels sont en effet le fruit de l'intelligence humaine et des rapports entretenus avec l'environnement, ils appartiennent donc en propre à leurs détenteurs. De sorte que pour certains auteurs, les savoirs autochtones collectés et colligés dans des ouvrages ou des bases de données ne représenteraient que des artefacts de savoirs, pâles reflets des connaissances détenues par les communautés (Nadasdy 129-130; Agrawal 325-326).

Les communautés autochtones peuvent, en outre, être considérées au Canada et dans l'outre-mer français, comme des ordres juridiques distincts au sein de l'État, quand bien même elles ne seraient pas reconnues de cette manière par ce dernier. En effet, ces communautés ont, bien souvent, conservé leurs propres institutions et leurs propres règles d’organisation et de régulation sociale même si celles-ci demeurent, parfois, inconnues à l'extérieur des communautés. Elles

11. La problématique des savoirs divulgués par les communautés ou par des chercheurs et leur diffusion successive des savoirs constitue une situation beaucoup plus complexe. 
constituent, alors, ce que Sally Falk Moore définit comme des champs sociaux semi-autonomes capables de se doter de leur propre normativité (Griffiths 1 ; Macdonald 138). De cette manière, les communautés disposent dans la plupart des cas de leurs propres lois et protocoles coutumiers de gestion de leur patrimoine culturel immatériel, à l'intérieur et vers l'extérieur des communautés.

$\mathrm{Au}$ Canada, ces lois et protocoles communautaires ont fait l'objet de nombreuses études ethnographiques, si bien qu'il est possible dans certains cas d’en dresser un portrait quasi complet (Thom et Bain). Il est important de noter que les lois et protocoles communautaires peuvent différer d'une communauté à l'autre. Ainsi, comme le note Brian Thom, chez les collectivités Kwakwaka'wakw (basées sur la côte Nord-ouest du Canada) :

Les protocoles coutumiers liés à des biens immatériels ont été utilisés pour la transmission de titres héréditaires ainsi que dans le cadre d'expressions et de prérogatives connexes telles que les légendes, les récits historiques, les danses, les performances, les dessins, les privilèges cérémoniels et certaines paroles rattachées à des rituels. Même s'ils appartiennent à des groupes familiaux descendant d'un ancêtre commun, ces modes dexpression culturelle traditionnelle peuvent être mis en pratique dans certaines circonstances par des membres reconnus. Certains noms, emblèmes et récits historiques traditionnels appartiennent également à des familles qui sont les seules à pouvoir les utiliser et les mettre en valeur. (Thom et Bain, i)

À l'inverse, chez les Inuits :

Les protocoles coutumiers [...] liés aux biens immatériels sont moins intégrés aux questions de structures et de statuts sociaux puisqu'ils découlent de la connaissance ultra-spécialisée secrète et personnelle nécessaire à la survie dans la région arctique. Certaines connaissances se rapportant à la quête de nourriture, une série de mots "magiques", de chants et de prières, la connaissance chamaniste et le dessin de vêtements constituent tous des aspects des biens immatériels des Inuits. Tous ces éléments, à l'exception du dessin de vêtements sont détenus par des personnes et peuvent être légués comme héritage ou vendus. Ils procurent des droits exclusifs qui peuvent enrichir des familles et en protéger d'autres contre les préjudices pouvant provenir du monde naturel ou spirituel. (Ibid., ii)

Dans d'autres cas, et notamment dans l'outre-mer français, les règles de gestion des savoirs autochtones, ne sont pas documentées précisément et il est, donc, plus difficile de les appréhender. On sait, néanmoins, que des règles existent en termes de circulation des savoirs, et notamment des tabous, des règles concernant le partage des savoirs au sein des communautés ou encore des processus de transmission ritualisés (Mwà Véé 13-15).

Ces règles, développées par les communautés, constituent le droit commun des populations pour la gestion de leur patrimoine culturel immatériel. Il s'agit 
là d'une interprétation de la normativité autochtone qui est souvent négligée car ces lois et ces protocoles ne sont pas, en général, codifiés ni reconnus par l'État. Cependant, par mesure de précaution et en vue du respect des communautés et de leur organisation, les chercheurs ou toute personne intéressée par travailler sur ou avec les savoirs autochtones, devraient prendre pour acquis que chaque communauté dispose de son propre système de gestion du patrimoine culturel immatériel et, donc, de règles relatives à leur diffusion à l'intérieur des communautés, mais aussi parfois vers l'extérieur.

Il faut, néanmoins, souligner que ces règles ne sont pas nécessairement adaptées dans le cadre de la rencontre entre les systèmes de gestion autochtones et le système occidental de gestion de la connaissance. En effet, les différences d'approches en termes de diffusion, de possibilités d'appropriation privative ou encore de valorisation peuvent parfois placer les systèmes de gestion autochtones dans une situation déséquilibrée susceptible de conduire à des abus. En effet, certaines communautés peuvent être particulièrement ouvertes au partage et à la diffusion libres de leurs savoirs, reste que ces derniers, intégrés au système occidental, peuvent se voir ensuite appropriés de manière privative et générer des bénéfices pour seulement certains individus et à l'exclusion des communautés (Burelli 2013 : 135-154).

Comme nous le verrons plus loin, ces lois et protocoles ont parfois évolué (plus récemment) vers des cadres éthiques modernes, adoptés par certaines communautés, notamment au Canada.

\section{La mise en place de cadres éthiques et de protocoles de recherche}

Il est possible d’observer au Canada, lémergence de nombreux cadres éthiques traitant de la problématique des savoirs autochtones, élaborés tant par les acteurs de la recherche que par les communautés autochtones. Il s'agit là d'un phénomène qui n'est pas observable en France. Des instituts de recherche comme l'IRD (Institut de Recherche pour le Développement) disposent bien de leur propre cadre éthique, mais ce dernier n'aborde pas la question spécifique du patrimoine culturel immatériel autochtone (Comité consultatif de déontologie et d'éthique, 2012).

\section{Les cadres éthiques académiques}

Il existe, au Canada, un énoncé de politique adopté par les trois Conseils de recherche canadiens (le Conseil de recherches en sciences humaines du Canada - CRSH, le Conseil de recherches en sciences naturelles et en génie du Canada - CRSNG, et les Instituts de recherche en santé du Canada - IRSC). Cet énoncé de politique concerne toutes les recherches impliquant des êtres humains, qu'il 
s'agisse de travaux dans le champ des sciences sociales (réalisation d'entretiens compréhensifs par exemple) ou dans celui des sciences médicales.

Ainsi les chercheurs et les universités percevant des financements de l'un ou plusieurs des trois Conseils sont tenus de respecter les principes éthiques de cet énoncé particulièrement détaillé ${ }^{12}$.

En ce qui concerne les savoirs autochtones l'énoncé propose une définition très détaillée de la notion de connaissances traditionnelles ${ }^{13}$ :

[Connaissances (savoirs)] "détenues par les peuples des Premières nations, les Inuits et les Métis qui constituent les peuples autochtones au Canada. Ces connaissances se rattachent à un lieu précis, sont généralement transmises oralement, et sont ancrées dans l'expérience de plusieurs générations. Ce sont les terres, le milieu, la région, la culture et la langue d'une communauté autochtone qui les déterminent. Les peuples autochtones présentent généralement les connaissances traditionnelles comme étant holistiques et faisant intervenir tout à la fois le corps, l'intelligence, les sentiments et l'esprit. Ces connaissances peuvent s'exprimer dans les symboles, les arts, les rites cérémoniels et pratiques usuelles, les récits et surtout les relations qui sétablissent. Le mot " tradition" ne signifie pas nécessairement " figé dans le temps». [...] Elles comprennent les connaissances préservées des générations passées de même que les innovations et nouvelles connaissances transmises aux générations suivantes. (Énoncé de politique des trois Conseils : Éthique de la recherche avec des êtres humains 120)

L'énoncé prévoit, en outre, que les chercheurs sont tenus de respecter «l'autorité gouvernementale " (Ibid. 128) des autochtones mais, aussi, de s'informer et de respecter les coutumes et les codes de pratique de la recherche qui s'appliquent à chacune des communautés visées par leur projet de recherche (Ibid. 132). En conséquence de quoi, alors que le droit canadien ne reconnaît pas nécessairement le droit coutumier autochtone non plus que les autorités de gouvernance autochtones ${ }^{14}$, les chercheurs se voient contraints, au travers des trois Conseils de prendre des mesures pour connaître (ils ne peuvent pas opposer l'oralité comme justification à leur méconnaissance) et respecter le droit coutumier, mais aussi les cadres modernes développés par les communautés dont nous parlerons dans la prochaine section.

12. C'est le cas notamment de l'auteur de ces lignes.

13. La notion de connaissances traditionnelles n'est pas définie ailleurs en droit canadien. 14. Tous les peuples autochtones canadiens ne bénéficient pas d'une reconnaissance de droits fonciers et de droits à l'autonomie gouvernementale. Certains peuples autochtones ont négocié, à partir des années 1970, des accords de revendications territoriales avec le gouvernement fédéral et les provinces du Canada. En ce qui concerne la reconnaissance des droits des peuples autochtones au Canada, voir l'ouvrage de Sébastien Grammond (Grammond 2013). 
Ces obligations disposent d'une force normative certaine étant donné que l'obtention et le versement de fonds de la part des trois Conseils sont conditionnés par leur acceptation et leur respect durant toute la durée des projets de recherche. Les chercheurs doivent ainsi, obtenir, avant de se rendre sur leurs terrains, un certificat d'éthique délivré au sein de leurs universités, et prévoir un formulaire de consentement qui devra être signé par chaque personne participant à la recherche ${ }^{15}$. Faute de certificat, les données et les résultats ne seront pas publiables. Les trois Conseils se montrent, en outre, très stricts avec le respect de lénoncé et peuvent suspendre et/ou ne pas renouveler, par la suite, un financement (voir Cadre de référence des trois organismes sur la conduite responsable de la recherche).

Certaines universités ont, elles aussi, développé leur propre cadre éthique à destination de leurs chercheurs. C'est le cas par exemple de l'université de Victoria en Colombie-Britannique qui a adopté en 2003 un document intitulé "Protocols and Principles for Conducting Research in an Indigenous Context " (University of Victoria Faculty of Human and Social Development).

Ce document apparaît, à bien des égards, particulièrement innovant au regard des règles classiques de la propriété intellectuelle et notamment en ce qu'il reconnaît un pouvoir de contrôle absolu aux communautés autochtones sur les données collectées durant la recherche. Un pouvoir qui peut les conduire à bloquer la publication de résultats de recherche :

The people participating have control over the results of the research process and as such have an absolute right to exercise control over the information they have volunteered. This includes the right to control it, to restrict access to it, or to withdraw part or all of the information from the actual research project findings. (Ibid. 5)

Ces principes prévoient aussi la mise en œuvre d'un partage des avantages lorsque cela est possible et approprié : "Researchers will not exploit informants, or the information gathered from the research, for personal gain or aggrandizement. Where possible and appropriate, fair return should be given for participants' help and services, which should be acknowledged in the final output" (Ibid. 6).

Ces différents cadres constituent autant de tentatives de refondation, à l'initiative du monde académique ${ }^{16}$, des relations entre la communauté des chercheurs et les communautés autochtones. Ils démontrent la capacité dévolution des scientifiques et de leurs institutions, et surtout leur capacité

15. Voir par exemple le bureau d'éthique et d'intégrité de la recherche de l'Université d'Ottawa : http://www.recherche.uottawa.ca/deontologie/, page consultée le 10 octobre 2014 16. Dans certains cas avec la consultation et/ou la participation des communautés autochtones. C’est le cas en particulier de l'Énoncé de politique des trois Conseils (Nickels et Knotsch 60). 
critique vis-à-vis des pratiques antérieures ${ }^{17}$. De leur côté, les communautés autochtones, ne sont pas en reste et ont aussi développé de nombreux cadres éthiques au Canada.

\section{Les cadres éthiques à l'initiative des communautés autochtones}

Les communautés autochtones à travers l'ensemble du Canada, ont aussi développé, depuis les années 1990 des codes ou des protocoles visant à encadrer les recherches les concernant. En voici une liste non exhaustive démontrant leur diversité et surtout leur multiplication au fil des ans :

- Dene Cultural Institute Guidelines (1991) ${ }^{18}$

- Council of Yukon First Nations (2000): Traditional Knowledge Research Guidelines: A Guide for Researchers in the Yukon ${ }^{19}$

- Manitoba First Nations Youth Council (2002): Traditional First Nations Code of Ethics ${ }^{20}$

- Gwich'in Tribal Council (2004): Traditional Knowledge Policy ${ }^{21}$

- Assemblée des Premières Nations du Québec et du Labrador (2005) :

Protocole de recherche des premières nations du Québec et du Labrador ${ }^{22}$

- Assembly of Nova Scotia Mi'kmaq Chiefs (2007): Mi'kmaq Ecological Knowledge Study Protocol ${ }^{23}$

17. «Ce sont principalement des chercheurs non autochtones qui ont défini et réalisé les projets de recherche visant les Autochtones ou les peuples autochtones au Canada. Les méthodes qu'ils ont utilisées ne prenaient généralement pas en compte la conception du monde qu'ont les Autochtones, et les projets de recherche nétaient pas forcément bénéfiques aux Autochtones ou aux communautés autochtones. C'est pourquoi les Autochtones voient encore la recherche, surtout la recherche qui ne provient pas de leurs communautés, d'un œil méfiant ou anxieux » (Énoncé de politique des trois Conseils : Éthique de la recherche avec des êtres humains 117).

18. Disponible à l'adresse suivante : http://www.wipo.int/export/sites/www/tk/en/databases/creative_heritage/docs/dci_guidelines.pdf, page consultée le 10 octobre 2014.

19. Disponible à l'adresse suivante : http://www.northerncontaminants.ca/done/guidelines/tkGuidelines/TK\%20Guidelines.pdf, page consultée le 10 octobre 2014. »

20. Disponible à l'adresse suivante : https://www.umanitoba.ca/student/asc/media/ Pamphlet_02a.pdf, page consultée le 10 octobre 2014.

21. Disponible à l'adresse suivante : http://www.gwichin.ca/TheGwichin/GTCTKPolicy.pdf, page consultée le 10 octobre 2014.

22. Disponible à l'adresse suivante : http://www.cssspnql.com/docs/centre-de-documentation/protocole-de-recherche---synthese.pdf?sfvrsn=2, page consultée le 10 octobre 2014. 23. Disponible à l'adresse suivante $:$ http://mikmaqrights.com/uploads/KMKMEK.pdf, page consultée le 10 octobre 2014. 
- Inuit Tapiriit Kanatami and Nunavut Research Institute (2007): Negotiating Research Relationships with Inuit Communities ${ }^{24}$.

Ces codes ou protocoles sont tous consultables en ligne et constituent des moyens pour les chercheurs d'appréhender les valeurs et les attentes des communautés autochtones. Ils ne sont néanmoins pas homogènes et de grandes différences d'approches sont parfois observables. Ainsi certains codes ou guides sont très détaillés et décrivent avec une grande précision les différentes étapes de la recherche avec les communautés (par exemple le guide Dene, la politique Gwich'in ou encore le protocole Mi'kmaq), alors que d'autres se concentrent plus sur les valeurs des communautés et leurs façons de concevoir les recherches (par exemple le code d'éthique des premières nations du Manitoba et le protocole de recherche des premières nations du Québec et du Labrador).

Ces cadres partagent néanmoins des principes et notamment : 1. la reconnaissance de la propriété sur le patrimoine culturel immatériel ; 2 . le respect du consentement préalable des communautés avant le début des recherches; 3 . le droit de participer aux recherches et au processus de décision ; 4. un droit de veto sur les résultats de la recherche et ou l'accès aux savoirs (Bannister 287).

Ces dispositifs font partie des codes et protocoles de recherche que les chercheurs doivent respecter au Canada dans le cadre de l'énoncé des trois Conseils. Ils sont aussi la preuve de la capacité des communautés à développer ellesmêmes un cadre normatif visant au respect de leurs valeurs et de leur patrimoine mais aussi et surtout la capacité à faire accepter ces cadres et à les transformer en normes juridiquement contraignantes pour les chercheurs.

En outre, ces différents codes et protocoles servent souvent de fondation au développement de pratiques contractuelles entre les autochtones et les chercheurs même si comme nous allons le voir ces pratiques peuvent aussi se développer en dehors de règles éthiques définies a priori.

\section{Le développement de pratiques contractuelles : le cas de la Station Gump en Polynésie française}

La Polynésie française est un territoire qui, du fait de ses importantes ressources, abrite plusieurs centres de recherche travaillant dans les domaines de la biologie, de la chimie et de l'environnement au sens large. Parmi ces organismes figurent notamment l'Université de Berkeley et sa Station Gump basée à Moorea.

24. Disponible à l'adresse suivante : https://www.itk.ca/system/files_force/Negotitiating-Research-Relationships-Researchers-Guide_0.pdf?download=1, page consultée le 10 octobre 2014. 
Cet organisme constitue un cas emblématique de développement de liens et de création de mécanismes déchange et de partage des résultats de recherche avec les autorités locales ainsi qu'avec la société civile de la Polynésie française.

Depuis l'installation en 1975 de la Station Gump en Polynésie française, l'Université de Berkeley a conclu plusieurs « General Agreements for Cooperation » successifs avec le gouvernement du territoire mais aussi avec les communautés autochtones de l'île de Moorea. Ainsi un accord-cadre a été signé entre l'Université de Berkeley et l'association « Te pu Atitia » en 2002 puis renouvelé en $2008^{25}$. Cette association a " pour objet de faciliter la transmission de l'héritage culturel à l'enfant, de conduire des programmes éducatifs, sportifs de loisirs et jeunesse se rapportant à l'environnement, à la science, la culture et les arts traditionnels ${ }^{26} »$. Pour cela elle s'est notamment donnée pour mission de collecter les éléments de la culture orale, de réclamer la restitution des biens culturels et de veiller à leur préservation ainsi qu'au respect du consentement préalable dans le cadre de leur diffusion ${ }^{27}$. En outre, bien qu'empruntant les formes d'une association, ses fondateurs ont prévu la présence d'un conseil des sages ou Tomite Toohitu, structure traditionnelle polynésienne, chargée de guider le bureau et de participer aux activités de l'association ${ }^{28}$.

La Station Gump accueille cette association afin de permettre une meilleure médiation entre les chercheurs et la société civile de Moorea. L'accord signé entre les deux parties prévoit une coopération dans plusieurs domaines :

Compilation of scientific and traditional knowledge of Moorea's terrestrial, aquatic, and marine ecosystems; Documentation and preservation of the ancestral heritage and culture of Moorea; Integration of the scientific method and traditional practices to generate new insights concerning the management, restoration and conservation of Moorea's natural and cultural patrimony; Dissemination of Polynesia's scientific and traditional knowledge to communities in Polynesia, California and the rest of the world via the internet. (General Agreement for Cooperation between the Association "Te Pu Atitia's" and the University of California 2)

Laccord prévoit, ainsi, une coopération sous plusieurs formes :

exchanges of information (with appropriate recognition being given to the author of the knowledge in any publications); Cooperation of the University faculty and students with members of the Association in mutually defined edu-

25. Voir http://www.moorea.berkeley.edu/outreach/atitia/, page consultée le 10 octobre 2014. 26. Article 2 des statuts de l'association consultable à l'adresse suivante : www.atiatia. over-blog.com

27. Ibid.

28. Article 8, Ibid. 
cational projects; Mutual logistical support during ongoing educational programs; Exchange in training and technical assistance of personal between the University and the establishment of joint seminars and workshops. (2)

Conscient des problématiques en termes de droits de propriété intellectuelle et de partage des résultats, l'accord-cadre prévoit que des accords spécifiques seront conclus afin de définir plus précisément les droits de propriété intellectuelle et les modalités de publications et d'exploitation des résultats (Ibid. 3).

Le "centre Atitia " est ainsi accueilli physiquement sur la propriété de l'Université de Berkeley. Il comprend un jardin ethnobotanique, un Fare pote'e (lieu de rassemblement traditionnel tahitien) et un quai donnant sur une réserve marine ${ }^{29}$. Cette proximité et cette présence au sein de la Station Gump du centre Atitia permettent d'initier et d'encourager une meilleure médiation entre les scientifiques du centre et la société civile. Au travers du centre Atitia, la Station Gump et l'association $\mathrm{Te} \mathrm{Pu}$ Atitia poursuivent en effet des programmes éducatifs et de recherche en commun se focalisant sur la biodiversité marine et terrestre, les savoirs traditionnels, la culture et les relations entre les sociétés humaines et les écosystèmes ${ }^{30}$ avec pour but commun : la documentation, la promotion et la préservation de l'héritage bioculturel polynésien ${ }^{31}$.

Le centre Atitia constitue, de ce fait, un excellent forum favorisant les interactions et les échanges, permettant aux étudiants en échange et aux chercheurs d'apprendre des experts locaux et vice-versa, de construire et d'étendre leurs connaissances ensemble.

Les pratiques initiées et développées par l'Université de Berkeley apparaissent comme tendant vers une véritable tentative de mise en relation des savoirs autochtones et des savoirs scientifiques dans des conditions de respect mutuel. Elles apparaissent d'autant plus intéressantes qu'elles reposent sur l'implantation ancienne et pérenne de l'Université de Berkeley ce qui a favorisé une acculturation progressive des communautés autochtones et des scientifiques.

\section{La mise en place d'institutions de conservation et de gestion}

Il arrive que des pratiques beaucoup plus complexes et globales émergent à l'image d'institutions de conservation et de gestion comme l'Agence de Développement de la Culture Kanak en Nouvelle-Calédonie (ADCK).

29. www.moorea.berkeley.edu/outreach/atitia/

30. Voir en ce sens le projet Biocode : http://mooreabiocode.org/

31. www.moorea.berkeley.edu/outreach/atitia/ 
C'est à l'issue des accords de Matignon que cette institution voit le jour à la demande de Jean-Marie Tjibaou ${ }^{32}$. Il s'agit d'un établissement public ${ }^{33}$ dont les missions consistent à : " valoriser le patrimoine archéologique et linguistique kanak; encourager les formes contemporaines d'expression de la culture kanak, en particulier dans les domaines artisanal, audiovisuel et artistique ; promouvoir les échanges culturels, notamment dans la région Pacifique Sud; et définir et conduire des programmes de recherche ${ }^{34}$ ».

Dans cette perspective, depuis 2002, l'ADCK et plus précisément le Département recherche et patrimoine (DRP), dirigé par Emmanuel Tjibaou, a mis en place un programme de collecte et de conservation du patrimoine culturel immatériel kanak au sens large (danses, tradition, littérature orale, savoirs écologiques) à l'échelle des 8 aires coutumières de l'île (Mwà Véé 11).

Pour cela l'ADCK travaille en étroite collaboration avec les conseils coutumiers et forment des collecteurs kanak. Ces derniers recueillent le patrimoine oral kanak en respectant un code éthique s'inspirant notamment des pratiques développées par les voisins de la République de Vanuatu (Mwà Véé 9-10). L'ADCK reçoit aussi, et spontanément, des savoirs que leurs détenteurs ne désirent pas transmettre aux jeunes ou qui ne le peuvent pas et souhaitent que l'ADCK en soit le dépositaire.

L'ADCK joue un rôle majeur de conservation en numérisant et en stockant le patrimoine kanak, mais aussi un rôle de gestion et de diffusion. Elle procède à la transmission du savoir notamment des danses traditionnelles lors dévènements culturels. Elle a aussi mis en place une échelle d'accès selon les savoirs collectés et la volonté de leur détenteur. Ainsi certains sont libres d’accès, alors que d'autres ne peuvent être communiqués sans l'accord de leur détenteur (Mwà Véé 8-9).

32. «Enfin, pour permettre l'expression et l'épanouissement sous toutes ses formes de la personnalité mélanésienne, une action soutenue sera mise en œuvre pour assurer l'accès de tous à l'information et à la culture. À cet effet, il sera créé un établissement public, dénommé « Agence de développement de la culture canaque ». Accords de Matignon-Oudinot du 26 juin 1988 ; Article $\mathrm{n}^{\circ} 93$ de la loi $\mathrm{n}^{\circ} 88-1028$ du 9 novembre 1988 portant dispositions statutaires et préparatoires à l'autodétermination de la Nouvelle-Calédonie en 1998. 33. Létablissement public a été transféré le $1^{\text {er }}$ janvier 2012 à la Nouvelle-Calédonie : Décret n 2011-1588 du 17 novembre 2011 portant transfert de l'Agence de développement de la culture kanak à la Nouvelle-Calédonie, JORF (Journal Officiel de la République Française) no 0269 du 20 novembre 2011, p. 19523.

34. http://www.adck.nc/presentation/le-centre-culturel-tjibaou/presentation, page consultée le 10 octobre 2014 ; Voir également les missions de l'ADCK définies dans l'Article 1, Décret no 89-524 du 27 juillet 1989 relatif à l'Agence de développement de la culture kanak, JORF du 28 juillet 1989, p. 9478. 
L'ADCK agit donc comme une véritable bibliothèque collectant et conservant les savoirs autochtones. Elle n'agit pas encore comme un organisme à l'interface des communautés autochtones et des utilisateurs potentiels de savoirs dans la mesure où telle n'est pas sa mission. De la même manière, elle n'est pas à l'image de la bibliothèque indienne (la Traditional Knowledge Digital Library) ${ }^{35}$ ou de l'institution péruvienne ${ }^{36}$ un organisme qui agit dans le domaine de propriété intellectuelle pour s'opposer par exemple au dépôt de brevets.

Cette institution a, néanmoins, fortement inspiré le projet de loi du pays ${ }^{37}$ relatif à la sauvegarde du patrimoine immatériel autochtone qui a vu le jour en 2010. Ce projet, en se basant sur le modèle de l'ADCK, propose la création d'une autorité administrative indépendante chargée de collecter, conserver et servir d'interface entre les chercheurs et les détenteurs autochtones (Burelli 2012b : 115-129).

Ces pratiques basées sur la constitution de bases de données et de définitions de règles d'accès peuvent être observées de plus en plus dans différents endroits du monde, qu'il s'agisse d'initiatives à léchelle de projets de recherche spécifiques $^{38}$ ou de programmes plus globaux comme celui mené par l'ADCK.

Tous ces projets semblent démontrer un fort besoin de conservation du patrimoine culturel immatériel autochtone vis-à-vis, notamment, des problèmes dérosion et de difficulté de transmission aux jeunes générations, mais aussi de normalisation des règles d'accès, d'utilisation et de partage des avantages. Dans cette perspective, les autochtones et les chercheurs sont particulièrement aidés par l'essor des moyens technologiques qui ont permis le développement d'outils de conservation et de gestion flexibles et aux capacités de stockage quasi-illimitées. Cependant la multiplication de ces outils et des bases de données ne va pas sans poser quelques problèmes quant aux méthodes de sélection et de stockage

35. En Inde, l'État a référencé les savoirs considérés comme appartenant au domaine public au sein d'une base de données dont la finalité est dềtre rendue accessible aux différentes autorités chargées de la délivrance de droits de propriété dans le monde et ce, le cas échéant, afin de mettre en évidence une absence de nouveauté ou d’activité inventive, étant donné l'existence de savoirs traditionnels antérieurs et publics. La bibliothèque est disponible en ligne à l’adresse : http://www.tkdl.res.in/tkdl/langdefault/common/Home. asp?GL=Eng, page consultée le 10 octobre 2014.

36. Le Pérou a, lui, adopté un cadre légal spécifique pour la protection des savoirs traditionnels. Plusieurs bases de données ont été créées afin de recueillir les savoirs traditionnels en fonction de leur degré de diffusion à l'extérieur des communautés. Le dispositif est géré par l'INDECOPI (Instituto Nacional de Defensa de la Competencia y de la Protección de la Propiedad Intelectual) qui tient compte de l'existence des savoirs traditionnels dans le cadre de l'analyse des demandes de droits de propriété intellectuelle (Filoche 2009).

37. C'est-à-dire voté par le congrès de la Nouvelle-Calédonie.

38. Voir par exemple le Projet Eloka (Exchange for Local Observations and Knowledge of the Arctic) en ligne à l'adresse : http://eloka-arctic.org/, page consultée le 10 octobre 2014. 
des savoirs mais aussi en termes de régime d'accès et d'utilisation post-collecte (Agrawal 325-336).

Il s'agit d’outils dont l'usage semble néanmoins appelé à se développer énormément, c'est pourquoi il est particulièrement important de suivre leur évolution et d'en évaluer l'utilisation.

\section{Conclusion}

Il n'est pas correct, aujourd'hui, de déduire de l'inaction des États en matière de réglementation du patrimoine culturel immatériel autochtone l'existence d'un vide juridique ni en France et ni au Canada. En effet, comme nous avons tenté de le montrer, il existe dans ces deux États de nombreuses pratiques et stratégies qui visent, ou qui intéressent, la régulation de la circulation des savoirs autochtones. Cette constatation d'un foisonnement normatif plutôt que d'un vide juridique a des conséquences très importantes pour les États et les acteurs sociaux.

Elle devrait d'abord encourager les États à ne pas focaliser leur attention autant qu'ils le font sur le droit international. Il semble que nous ayons affaire dans ce domaine à une quasi-saturation des débats et du droit tant les évolutions récentes (notamment le protocole de Nagoya) démontrent que la marge d'amélioration des concepts et des principes devient de plus en plus faible (Burelli 2012a : 6062). Ainsi il nous apparait que, aujourd'hui, le nœud du problème se situe plutôt au niveau national. En effet, le droit des Autochtones constitue encore un enjeu particulièrement sensible pour beaucoup d'États, et la notion d'Autochtones et la question de leurs droits restent très contestées au niveau étatique ${ }^{39}$. Le foisonnement des pratiques mis en avant devrait aussi pousser les États à plus de réflexion quant aux initiatives mises en œuvre localement avant même d'envisager la transposition des principes de droit international. En effet, l'inaction des États, qui perdure parfois depuis les années 1990, a encouragé dans certains cas le développement de pratiques innovantes. Des expériences qui pourraient informer les États sur la pertinence d’agir, mais qui pourraient aussi servir de sources d'inspiration à propos d’outils ou de principes à généraliser.

La diversité des pratiques que nous avons présentées pourrait aussi servir à convaincre les acteurs sociaux concernés (et en particulier les communautés autochtones et les chercheurs) que l'inaction des États n'est pas une fatalité, mais qu'au contraire, il est possible d'imaginer des alternatives originales. Dans

39. Par exemple, la France ne reconnaît que timidement, et souvent de manière indirecte, que des populations autochtones sont présentes sur son territoire. Le Canada, quant à lui, n'a pas encore reconnu de titre aborigène (même si la Cour suprême du Canada en a reconnu le principe en 1997 dans l'affaire Delgamuukw c. Colombie-Britannique [1997] 3 R.C.S. 1010). 
cette perspective, l'attitude a priori défaillante des États pourrait finalement se révéler une opportunité plutôt qu'une fatalité. En effet elle laisserait aux individus et/ou à leurs institutions la liberté de créer des cadres pour leur collaboration. Des cadres qui pourraient le cas échéant être potentiellement plus adaptés à leurs attentes que s'ils avaient été développés par les administrations centrales. Il faut néanmoins se garder de tout angélisme quant à la capacité des communautés autochtones et des chercheurs à réinventer et à refonder leurs relations expurgées de tout rapport de pouvoir ${ }^{40}$, en particulier dans un contexte postcolonial. Il n'en demeure pas moins que l'inaction des États ouvre cette possibilité, sinon cette responsabilité, pour les communautés autochtones et scientifiques qui ont de nombreux intérêts communs dans la recherche.

\section{Bibliographie}

AGRAWAL, Arun. "Classification des savoirs autochtones : la dimension politique ». Revue Internationale des Sciences Sociales 173:3 (2002) : 325-336.

Assemblée des Premières Nations du Québec et du Labrador. Protocole de recherche des premières nations du Québec et du Labrador. 2005. Disponible à l'adresse : http://www.cssspnql.com/docs/centre-de-documentation/protocole-de-recherche--synthese.pdf?sfvrsn=2, page consultée le 10 octobre 2014.

Assembly of Nova Scotia Mi'kmaq Chiefs. Mi'kmaq Ecological Knowledge Study Protocol. 2007. Disponible à l'adresse : http://mikmaqrights.com/uploads/ KMKMEK.pdf, page consultée le 10 octobre 2014.

BANNISTER, Kelly. "Non-legal instruments for the protection of intangible cultural heritage: Key roles for ethical Codes and community protocols." Bell, C. E., ed. Protection of First Nations cultural heritage: laws, policy, and reform. Vancouver, BC: UBC Press, 2009. 278-309.

BERKES, Fikret. Sacred Ecology. Traditional Ecological Knowledge and Resource Management. Philadelphia: Taylor \& Francis, 1999.

BURELLI, Thomas. "Collaboration entre scientifiques et communautés autochtones et locales : Le patrimoine culturel immatériel autochtone face aux logiques de développement et de valorisation de l'innovation ». Gaumont-Prat, H. (sous la dir. de). Innovation et Droit: Actualités de la jurisprudence en matière de propriété industrielle. Paris, coll. «Grands Colloques », L.G.D.J. (Librairie générale de droit et de jurisprudence), 2013. 135-154.

40. Macdonald, notamment, reconnaît que le pluralisme n’est pas nécessairement lié à des solutions plus démocratiques et égalitaires : « Toutefois, la vie inter- et intrasubjective nest ni radicalement démocratique ni radicalement égalitaire. Je ne prétends pas que l'hypothèse du pluralisme, soit l'idée que les sujets de droit sont également ceux qui façonnent le droit, nous permette comme société de surmonter la discrimination, l'abus du pouvoir et les conflits interculturels » (Macdonald 143). 
" Faut-il se réjouir de l'adoption du protocole de Nagoya ?». Revue Juridique de l'Environnement 1 : (2012a) : 45-62.

. «Propriété intellectuelle et savoirs traditionnels en Nouvelle-Calédonie - Pertinence et potentialités du projet de loi du pays relatif à la sauvegarde du patrimoine immatériel autochtone ». Faberon, J.-Y. et T. Mennesson (sous la dir. de), Peuple premier et cohésion sociale en Nouvelle-Calédonie - Identités et rééquilibrages. Marseille : Presses Universitaires d'Aix-Marseille, 2012b. 115-129. " La reconnaissance et la protection des savoirs traditionnels associés à la biodiversité dans l'outre-mer français : une nécessité jusqu’à présent négligée ", Les Cahiers d’anthropologie du droit, Le statut des peuples autochtones. $\grave{A}$ la croisée des savoirs, (2012c). 137-162.

Comité consultatif de déontologie et d'éthique. Guides des bonnes pratiques pour les recherches pour le développement. $2^{\mathrm{e}}$ édition. 2012. Disponible sur : http:// www.ird.fr/content/download/46083/353877/version/3/file/GBP2012consultation.pdf. Consulté le 20 décembre 2013.

Conseil de recherches en sciences humaines du Canada, Conseil de recherches en sciences naturelles et en génie du Canada, Instituts de recherche en santé du Canada. Cadre de référence des trois organismes sur la conduite responsable de la recherche. 2011. Disponible sur : http://www.rcr.ethics.gc.ca/_doc/FrameworkCadreReference_fra.pdf. Consulté le 20 décembre 2013.

- Énoncé de politique des trois Conseils : Éthique de la recherche avec des êtres humains. 2010. Disponible sur : http://www.ethics.gc.ca/pdf/fra/eptc2/ EPTC_2_FINALE_Web.pdf. Consulté le 20 décembre 2013.

Council of Yukon First Nations. Traditional Knowledge Research Guidelines: A Guide for Researchers in the Yukon. 2000. Disponible à l'adresse : http://www. northerncontaminants.ca/done/guidelines/tkGuidelines/TK\%20Guidelines.pdf, page consultée le 10 octobre 2014.

Dene Cultural Institute Guidelines, 1991. Disponible à l'adresse : l'adresse suivante : http://www.wipo.int/export/sites/www/tk/en/databases/creative_heritage/ docs/dci_guidelines.pdf, page consultée le 10 octobre 2014.

Environnement Canada. Accès aux ressources génétiques et partage des avantages découlant de leur utilisation au Canada: Possibilités d'une nouvelle orientation de politique. 2009. Disponible sur : http://www.biodivcanada.ca/1AB19CC4-9C19-44B6972B-42243654600B/accessing_genetic_f.pdf. Consulté le 20 décembre 2013.

---. L'accès et le partage des avantages des ressources génétiques : l'atelier d'experts sur le « Programme en S-T». 2005.

FILOCHE Geoffroy. «Les connaissances, innovations et pratiques traditionnelles en matière de biodiversité : un kaléidoscope juridique ", Droit et société, 72 (2009) : 433-458.

Fondation pour la Recherche sur la Biodiversité. «Pertinence et faisabilité de dispositifs d'accès et de partage des avantages en outre-mer, portant sur les 
ressources génétiques et les connaissances traditionnelles associées ». Études et documents du Commissariat général au développement durable 48 (2011). Disponible sur : www.developpement-durable.gouv.fr/IMG/pdf/ED48.pdf. Consulté le 20 décembre 2013.

General Agreement for Cooperation between the Association "Te Pu Atitia's" and the University of California. 2008. Disponible à l'adresse suivante : http://moorea. berkeley.edu/sites/default/files/u4/Atitia_Agreement_2008_En.pdf, page consultée le 10 octobre 2014.

Gouvernement du Canada. Atelier du Nord sur l'accès aux ressources génétiques et aux connaissances traditionnelles connexes et le partage des avantages. 2005.

Gouvernement du Canada et Gouvernement du Nouveau-Brunswick. Rapport d'atelier: Accès aux ressources génétiques forestières et partage des avantages, opportunités et défis potentiels pour les gouvernements et les parties concernées du secteur forestier. 2006. Disponible sur : http://publications.gc.ca/collections/ collection_2012/ec/En4-90-2008-fra.pdf. Consulté le 20 décembre 2013.

GRAMMOND, Sébastien. Terms of Coexistence: Indigenous Peoples and Canadian Law. Toronto: Carswell, 2013.

GRIFFITHS, John. "What is Legal Pluralism?" Journal of Legal Pluralism 24 (1986): 1-55.

Gwich'in Tribal Council. Traditional Knowledge Policy. 2004. Disponible à l'adresse : http://www.gwichin.ca/TheGwichin/GTCTKPolicy.pdf, page consultée le 10 octobre 2014.

Inuit Tapiriit Kanatami and Nunavut Research Institute. Negotiating Research Relationships with Inuit Communities. 2007. Disponible à l'adresse : https://www. itk.ca/system/files_force/Negotitiating-Research-Relationships-ResearchersGuide_0.pdf?download=1, page consultée le 10 octobre 2014.

MACDONALD, Roderick. "L'hypothèse du pluralisme juridique dans les sociétés démocratiques avancées ». R.D.U.S. (Revue de droit de l'Université de Sherbrooke) $33: 1-2$ (2002) : 135-152.

Manitoba First Nations Youth Council. Traditional First Nations Code of Ethics, 2002. Disponible à l'adresse : https://www.umanitoba.ca/student/asc/media/ Pamphlet_02a.pdf, page consultée le 10 octobre 2014.

Mwà Véé Revue culturelle kanak. «Du patrimoine immatériel aux archives kanak ». 50 (2005).

NADASDY, Paul. Hunters and Bureaucrats. Power, Knowledge, and AboriginalState Relations in the Southwest Yukon. Vancouver: UBC Press, 2003.

NICKELS, Scot \& Cathleen KNOTSCH. "Inuit perspectives on research ethics: The work of Inuit Nipingit." Etudes/Inuit/Studies 35:1-2 (2011): 57-81.

ROCHER, Guy. 1988. "Pour une sociologie des ordres juridiques ». Les cahiers du droit 29:1 (1998) : 91-120. 
SAMBUC, Henry Philippe. La protection internationale des savoirs traditionnels: la nouvelle frontière de la propriété intellectuelle. Paris, L'Harmattan, 2003.

SMITH, Linda Tuhiwai. Decolonizing Methodologies: Research and Indigenous Peoples. Second Edition. New York: Zed Books, 2012.

THOM, Brian \& Don BAIN. Aboriginal Intangible Property in Canada: An Ethnographic Review / Biens immatériels autochtones au Canada : une étude ethnographique. Ottawa Industry Canada, Marketplace Framework Policy Branch, 2004. Disponible sur : https:// www.ic.gc.ca/eic/site/ippd-dppi.nsf/vwapj/Thom_Final_Report_e_proofed_28feb05. pdf/\$FILE/Thom_Final_Report_e_proofed_28feb05.pdf et http://www.ic.gc.ca/eic/site/ ippd-dppi.nsf/fra/ip01204.html. Consulté le 11 septembre 2014.

University of Victoria Faculty of Human and Social Development. Protocols and principles for conducting research in an indigenous context. 2003. Disponible sur : http://web.uvic.ca/igov/uploads/pdf/Indigenous\%20Research\%20Protocols.pdf. Consulté le 20 décembre 2013.

Résumé : Les savoirs autochtones ont été progressivement reconnus au niveau international et notamment dans la Convention sur la Diversité Biologique adoptée lors de la Conférence de Rio en 1992, mais aussi dans de nombreux autres textes internationaux. Pourtant à ce jour très peu d'États sont parvenus à mettre en œuvre ces traités. Beaucoup font preuve aujourd'hui d'un immobilisme paradoxal compte tenu des efforts considérables déployés en termes de négociation au niveau international depuis 1992 (c'est le cas de la France et du Canada). Cet immobilisme n'est pas pour autant synonyme d'absence de régulation étant donné le dynamisme normatif infra-étatique dont font preuve en particulier les autochtones et les chercheurs. Il est en effet possible d'observer une grande diversité d'initiatives déployées pour l'encadrement de la circulation des savoirs traditionnels associés. Dans cet article nous proposons de présenter une série d'initiatives innovantes menées en France et au Canada.

Mots clés : Droit, éthique, peuples autochtones, patrimoine culturel immatériel, savoirs traditionnels, savoirs autochtones.

Summary: Traditional knowledge has been gradually recognized at the international level in particular in the Convention on Biological Diversity adopted during the Rio Conference held in 1992, but also in many other international texts. To date, however, very few countries have been able to implement these texts. This opposition to change at the national level appears to be very paradoxical given the fact that States have put a great deal of efforts in the negotiation process of international texts since 1992 (this is the case of France and Canada). However, this situation does not signify the existence of a legal vacuum. In fact, at a local level, indigenous people and researchers have developed several tools and strategies to regulate the circulation of traditional knowledge. In this article we propose to describe a series of innovative practices developed in France and in Canada.

Keywords: Law, ethic, indigenous people, intangible cultural heritage, traditional knowledge, indigenous knowledge.

Thomas Burelli est doctorant en droit à l'université d'Ottawa et assistant de recherche à la Chaire de recherche du Canada sur la diversité juridique et les peuples autochtones. Spécialité : Droit (droit de l'environnement, propriété intellectuelle, droit des peuples autochtones), anthropologie du droit.

Thomas Burelli, PhD candidate at the University of Ottawa - Research assistant in the Canada Research Chair on legal diversity and indigenous people. Law (environmental law, intellectual property, indigenous people law), legal anthropology. 